\title{
Full and Partial Grounding
}

\author{
Kelly Trogdon (Virginia Tech) and D. Gene Witmer (University of Florida) \\ Journal of the American Philosophical Association, forthcoming
}

Abstract: While controversy about the nature of grounding abounds, our focus is on a question for which a particular answer has attracted something like a consensus. The question concerns the relation between partial grounding and full grounding. The apparent consensus is that the former is to be defined in terms of the latter. We argue that the standard way of doing this faces a significant problem and that we ought to pursue the reverse project of defining full grounding in terms of partial grounding. The guiding idea behind the definition we propose is that full grounding is what happens when partial grounding works in a way that ensures that the grounded is nothing over and above the grounds. We ultimately understand this idea in terms of iterated nothing-over-and-above claims.

\section{Introduction}

Suppose that a collection of one or more facts $\Delta$ grounds some fact A. As a preliminary characterization of the distinction between partial and full grounding, we can say that $\Delta$ partially grounds $\mathrm{A}$ if it contributes to explaining $\mathrm{A}$, and $\Delta$ fully grounds A if nothing needs to be added to $\Delta$ to get a fully satisfactory explanation of A. As any ground contributes to explaining what it grounds, any ground is a partial ground. But not every partial ground provides a fully satisfactory explanation of what it grounds, so not every ground is a full ground. Let a merely partial ground be a partial ground that isn't a full ground.

This characterization serves to identify the distinction between partial and full grounding, but it leaves us in need of an account of the distinction-an account that exhibits this difference in explanatory import as due to a corresponding difference in the way the grounds are related to the grounded. There is a popular definition of partial grounding in terms of full grounding that seems to meet this desideratum. On that definition — call it the "standard definition"-a merely partial ground is one that 
leaves out at least one other ground needed to provide a full ground of the fact to be explained. If correct, the standard definition provides a tidy explanation of the difference in explanatory important between merely partial and full grounds.

As we have a pre-theoretic grip on both partial and full grounding, endorsing the standard definition means making a substantive claim; this isn't a mere stipulative matter. And there is a significant difficulty facing that definition. As we elaborate below, the standard definition rules out as impossible a certain kind of situation that otherwise seems possible. It might turn out that theoretical considerations ultimately favor the standard definition to such an extent that we should reject that appearance of possibility. But we can't assess this defense of the standard definition without comparing it to other options. It is important, then, both to get clear on the problem and on whether there is a viable alternative. It is worth stressing, further, that what we say about the relation between full and partial grounding is likely to have repercussions "downstream" in the theory of grounding. We should take care not to make a foundational error.

The paper contains three main sections. In $\$ 2$ we set out the standard definition and the difficulty it faces. We then consider alternatives that reverse the order of definition, taking partial grounding for granted and defining full grounding in terms thereof. In $\$ 3$ we consider a variety of unsuccessful attempts to implement this strategy. The failures prove instructive, however, and we develop and propose a definition in $\$ 4$. The proposed definition is guided by the idea that full grounding is what happens when partial grounding works in a way that ensures that the grounded is nothing over and above the grounds. We ultimately understand this idea in terms of iterated nothing-over-and-above claims.

\section{A difficulty with the standard definition}

Among those who have explicitly addressed the distinction between full and partial grounding, the following definition has emerged as a near consensus view:

$\Delta$ partially grounds $\mathrm{A}={ }_{\text {def. }} \Delta$, either on its own or together with other facts, fully grounds A. 
This is the standard definition alluded to above. ${ }^{1}$ It has not gone entirely unquestioned. Clark (2015: 190) raises a puzzle about the standard definition and emphasizes that rejecting it is genuine option, pointing out that the intuitive notion of partial grounding can “stand by itself'. Fine (2012) and Rosen (2015) consider a potential counterexample to the standard definition involving knowledge. And Leuenberger (2020) proposes two potential counterexamples, one involving alien properties and another totality facts. (We discuss the knowledge case and a counterexample akin to Leuenberger's second case below.) Still, the problem on which we focus seems to be at least underappreciated, and no alternative to the standard definition has yet emerged.

That problem comes into focus once we consider that the standard definition implies the following definition of merely partial grounding:

$\Delta$ merely partially grounds $\mathrm{A}={ }_{\text {def. }} \Delta$ does not fully ground $\mathrm{A}$, but $\Delta$ together with other facts fully grounds A.

Consider just the left-to-right implication of this definition: necessarily, if $\Delta$ merely partially grounds $\mathrm{A}$, then there is some collection of one or more facts $\Gamma$ such that $\Delta$, $\Gamma$ fully grounds $A$. If that is right, then a merely partial ground cannot exist without there being something else that, as we might put it, augments it so that together they provide a full ground. Call this the augmentation thesis. If the augmentation thesis is correct, then a certain kind of situation is impossible, namely, one in which some $\Delta$ merely partially grounds a fact $\mathrm{A}$, but there is no angmenting $\Gamma$ such that $\Delta$ together with $\Gamma$ fully grounds A.

Call any such situation a case of Non-Augmented (merely partial) Grounding—or, for short, a $N A G$ case. The difficulty with the standard definition is this: there is a significant case to be made for the possibility of NAG cases but no good reason,

${ }^{1}$ Proponents include Audi (2012: 698), Litland (2015: 484), Rosen (2010: 115), and Skiles (2015: 720). 
apart from an assumption of the standard definition itself, for thinking them impossible.

We will consider two types of NAG cases. In one sort, a fact A has a merely partial ground $\Delta$ where $\Delta$ cannot be combined with any other facts to provide a full ground of A because no collection of facts fully grounds A. In another, less simple kind of case, there is some collection of facts that fully grounds A, but the merely partial ground $\Delta$ cannot be combined with any other collection of facts to provide a full ground of A. We consider each in turn.

In the first sort of case, nothing is a full ground of the grounded fact. You might think that the thesis of metaphysical foundationalism - roughly, the view that every fact is either fundamental or fully grounded by fundamental facts_-rules out such cases. But let us say that a fact is weakly fundamental just in case it has no full grounds and strongly fundamental just in case it has neither full nor partial grounds (Leuenberger 2020). If metaphysical foundationalism is cast in terms of strongly fundamental facts, it indeed rules out this kind of NAG case; but if understood in terms of weakly fundamental facts, it does not. Of course, whether metaphysical foundationalism of either sort should be adopted is a contested matter, but it seems to us that extant arguments for foundationalism do not discriminate between the two versions. Consider, for example, Schaffer's reality inheritance argument (2016: 95). The argument proceeds roughly as follows: where there is full grounding there must be reality inheritance; where there is reality inheritance there must be a source of reality; and for something to be a source of reality is in part for it be fundamental. On the face of it, weakly fundamental facts can play the role of being a source of reality in the relevant sense.

There is precedent for taking NAG cases of the first type seriously. One way to make sense of "strong emergence" — as suggested (not endorsed) by Wilson (2018) — is to construe emergent facts in just this way. Emergent mental facts may be partly explained by physical facts that ground them even while they cannot be fully explained by such — or by any other kind of fact. While we do not mean here to defend the claim that any actual facts are emergent in this way, we think it is hard to 
see any reason that there couldn't be facts that have exactly that structure. (While Kim 2009 doubts the coherence of emergence, his argument notably overlooks grounding-theoretic characterizations of the notion.) The point has nothing to do with mentality in particular; there may be properties utterly unfamiliar to usuninstantiated in the actual world —-such that in some possible world facts about their instances are partially grounded in other kinds of facts but not fully grounded by anything.

We recognize that actual cases can be more persuasive than such mere possibilities. Are there any actual facts that have partial grounds but no full grounds? We suggest two candidate cases, the first of which is quite simple. Consider a simple atomic fact-some object a has property F. Suppose that this fact lacks full grounds, as a and $\mathrm{F}$ do not decompose into more basic objects and properties. There is some plausibility to the claim that this fact is nevertheless partially grounded by the fact that a exists. If this is right, then the fact that a is $\mathrm{F}$ is a NAG case. $^{2}$

The second case involves the interaction of quantified facts and singular facts. For convenience we will use "Q" in conjunction with other letters to name those of the first kind and "S" in conjunction with other letters to name those of the second kind. Consider some true restricted generalization:

\section{QFG: All Fs are Gs.}

Let this be an accidental truth as well. Interestingly, this kind of case has been used by both Chudnoff (2013: 182-187) and Skiles (2015: 729-736) to argue against the claim that full grounds always necessitate what they ground. But their observations can be turned to a different purpose. Suppose that there are exactly $n$ things that are F; let them be $a_{1}, a_{2}, \ldots a_{n}$. Consider the fact:

SG: $a_{1}$ is $G, a_{2}$ is $G, \ldots$ and $a_{n}$ is $G$.

\footnotetext{
2 Thanks to Kevin Mulligan for suggesting this case.
} 
It seems entirely plausible that SG partially grounds QFG. As Skiles notes, it obviously doesn't necessitate QFG. If one thinks that this collection is not only a partial ground but a full ground of QFG, then one should conclude that full grounds need not necessitate. But is it plausibly a full ground? No-not if a full ground is one that provides a fully satisfactory explanation of the grounded fact.

So far this looks nothing like a NAG case, as there is no reason yet to think we cannot find an augmenting ground to add to SG to get a full ground. Perhaps this will do:

QF: The only $F$ things are $a_{1}, a_{2}, \ldots a_{n}$.

(We understand QF to imply that every listed individual is $\mathrm{F}$ as well as that every other individual is not.) Why not augment SG with QF? The resulting collection specifies the only things that are $\mathrm{F}$ and then implies, of each of those things, that it is G; that would appear to be a natural enough candidate for a full ground of QFG. It is also, of course, a necessitating ground of QFG. Skiles considers this way of defending the claim that full grounds always necessitate but rejects it, noting that "...the fact that these are the only things that are F is itself an accidental generalization, one in need of a necessitating ground" (2015: 734). He seems then to think that QF must have some full ground of its own which necessitates it, if the necessitarian position is to be defended. But there is another option here: perhaps QF doesn't have a full ground. Skiles claims that it is "undesirable in the extreme" to say that this fact is fundamental (2015: 734) Skiles' rejection here is understandableat least, if we read "fundamental" as strongly fundamental, as having no grounds at all. After all, QF seems to be at least partially grounded by:

SF: $a_{1}$ is $F, a_{2}$ is $F, \ldots$ and $a_{n}$ is $F$.

So we agree that QF is not strongly fundamental. But unless we assume that NAG cases are impossible, why not allow that QF is partially grounded by SF without having any full grounds? 
One upshot of this discussion is that this argument against the claim that full grounds necessitates depends on ruling out NAG cases. More to the present point, however, is the observation that facts of the form of QF may count as genuine NAG cases.

That conclusion can be resisted. Can SF be augmented in some appropriate way? Say that all the non-F individuals that exist are $a_{n+1}, a_{n+2}, \ldots$ and $a_{n+m}$. Now consider two additional facts:

SNF: $a_{n+1}$ is not $F, a_{n+2}$ is not $F \ldots$ and $a_{n+m}$ is not $F$.

QE: For any $x, x=a_{1}$ or $x=a_{2}$, or $\ldots x=a_{n+m}$.

Perhaps SF, SNF and QE together provide a full ground for QF. This has some intuitive appeal: list the individuals that are $\mathrm{F}$, those that are not $\mathrm{F}$, and then say that those are all the individuals that exist. Then this may fully explain the fact that the only $\mathrm{F}$ things are $\mathrm{a}_{1}, \mathrm{a}_{2}, \ldots$ and $\mathrm{a}_{\mathrm{n}}$.

Perhaps. We need not take a stand on this question; just consider the two options. If SF, SNF, and QE do not fully ground QF, then it seems likely that we have a NAG case, as any other options for a full ground seem less plausible than this collection. If they do fully ground QF, then we should shift our attention to yet another general claim-this time, QE. What, if anything, grounds that fact?

Consider the fact that enumerates of each individual that it exists:

SE: $a_{1}$ exists, $a_{2}$ exists, $\ldots .$. and $a_{n+m}$ exists.

This collection is a partial ground of QE; it is not a full ground, however, as it obviously fails to provide a fully satisfactory explanation thereof. Might there be further grounds that augment SE to provide a full ground of QE? It seems unlikely. The pattern thus far is the familiar one of flattening the bump in the carpet in one location only to see it pop up elsewhere. Leuenberger (2020) arrives at a similar conclusion focusing on a totality claim about the existence of facts, using Armstrong's totaling relation (2004: 72). Of course, we have hardly proven that the 
augmentation thesis fails in one of these places, but the way in which generality persistently raises the specter of a NAG case should carry substantial weight. ${ }^{3}$

Let us now turn to the less simple kind of NAG case, namely, one in which some fact $\mathrm{A}$ has a full ground, $\Delta$ is a mere partial ground of $\mathrm{A}$, but no facts can be combined with $\Delta$ to form a full ground of $\mathrm{A}$. Might this happen? We may overlook this kind of case if we imagine that the members of $\Delta$ are among the facts included in the full ground of $\mathrm{A}$; but it is not obvious that they must be included. What might such a situation look like? Again, we have two candidate cases to consider, the first of which is modeled on Williamson's (2000) "Knowledge First" view according to which knowledge requires truth and belief but cannot be analyzed in terms of truth, belief, and additional conditions. Suppose that

KNOWLEDGE: Amy knows that humans have set foot on the moon.

is partially grounded by

TRUTH: It is true that humans have set foot on the moon.

According to this Grounding version of Knowledge First (GKF), nothing can be added to TRUTH to yield a full ground of KNOWLEDGE. As stipulated, the truth of GKF does not rule out there being full grounds for KNOWLEDGE. Suppose that there is some collection of facts concerning the ways in which Amy's neural states are causally connected to people who have been on the moon such that those facts fully ground KNOWLEDGE. Call that collection CAUSE. Suppose further that CAUSE does not include the fact that it is true that humans have set foot on the moon (the facts in CAUSE may imply this truth without including it). So, we cannot subtract TRUTH from CAUSE to get an augmenting ground. If GKF is true, then we may have

\footnotetext{
${ }^{3}$ Fine (2012) argues that it's useful for the purposes of developing an impure logic of ground to postulate a singular full ground for QE, one not partially grounded by identity facts (e.g. $\left[a_{1}=a_{1}\right]$ ). Nothing in Fine's discussion, however, suggests that SE (a collection of existence rather than identity facts) fails to partially ground this singular fact.
} 
a NAG case of this second kind, where the grounded fact is not even weakly fundamental.

(Fine's treatment of this case is subtle and requires special comment. He considers two different conceptions of the partial variety of so-called "strict" grounding. On the first conception — called "partial strict" grounding-any strict ground of the partial variety is either a full ground or can be augmented with some fact to get a full ground. On the second_- "strict partial" grounding- - he notes that this might not be the case. Hence, for Fine the idea that TRUTH is a non-augmented ground for KNOWLEDGE is a live option if we have strict partial but not partial strict grounding in mind. For what it's worth, we find Fine's notion of strict partial grounding hard to get a grip on, as it is defined in terms of what he calls "weak" grounding, a notion that itself is difficult to understand; see deRosset 2013a.)

For a second case, consider the following quantified facts:

DOUBLE: There exists some $\mathrm{x}$ such that $\mathrm{x}$ is $\mathrm{F}$ and $\mathrm{G}$.

SINGLE: There exists some $\mathrm{x}$ such that $\mathrm{x}$ is $\mathrm{F}$.

Suppose that a is both F and G. Call this fact INSTANCE. This fact doesn't include SINGLE and it fully grounds DOUBLE. If SINGLE partially grounds DOUBLE, then it appears to be a NAG case of the second sort. ${ }^{4}$

In the two cases discussed above, we have a mere partial ground for some fact, and a full ground for that fact that does not include the mere partial ground. Why not simply combine the mere partial grounds with the full grounds in these cases to get further full grounds? As for the first case, the thought would be to simply add CAUSE to TRUTH and the result would itself be a full ground of KNOWLEDGE. As for the second case, the thought is that we would get an additional full ground for DOUBLE simply by adding INSTANCE to SINGLE.

\footnotetext{
${ }^{4}$ Thanks to Ted Sider for suggesting this case.
} 
This maneuver, however, requires allowing a full ground to include facts that seem irrelevant. Intuitively, the collection that consists of everything in CAUSE as well as TRUTH is one which includes an irrelevant element, as the facts in CAUSE seem to do all the work on their own. The same goes for the collection consisting of INSTANCE and SINGLE— the former seems to do all the work on its own.

Audi (2012: 699) has proposed a kind of minimality requirement according to which any sub-collection of $\Delta$ must "actually do some work" with respect to making A obtain when $\Delta$ fully grounds A. As an unqualified ban on unnecessary elements, the requirement seems too strong, as argued by Werner (forthcoming). Werner himself proposes a more discriminating version, however, that operates at the level of immediate grounds. Roughly speaking, an immediate ground is one that needn't be mediated, and a ground has to be mediated when it can only ground the grounded via some intermediate grounding steps (Fine 2012). According to Werner's minimality principle, if $\Delta$ is an immediate full ground of $\mathrm{A}$, then either $\Delta$ is minimal or is itself the amalgamation of immediate full grounds each of which is itself minimal.

Returning to GFK, it seems that TRUTH is an immediate partial ground of KNOWLEDGE, and so long as there is some full ground of KNOWLEDGE, it has an immediate full ground. Suppose CAUSE is that immediate full ground. If we simply add CAUSE to TRUTH to get a full ground, that would also be immediate; yet it is not minimal, nor is it an amalgamation of full grounds each of which is minimal. Given Werner's minimality requirement, then TRUTH, CAUSE is not a full ground for KNOWLEDGE after all and our original suggestion that TRUTH is a partial ground without an augmenting ground has yet to be ruled out. Corresponding considerations apply to the case of SINGLE.

Between merely possible NAG cases (such as emergent facts), the two candidate cases of actual facts that are weakly fundamental, and the two candidate cases of facts just considered that are not even weakly fundamental but have non-augmented merely partial grounds, it seems clear that we should not rule out the possibility of 
NAG cases without at least considering alternatives to the standard definition. Let us turn to a search for an alternative.

\section{How not to define full grounding in terms of partial grounding}

What would an alternative look like? Presumably we should define partial grounding in terms of full grounding or vice versa. In this section we consider a number of inadequate attempts to define full grounding in terms of partial grounding.

In our preliminary characterization, we said that $\Delta$ is a full ground for $\mathrm{A}$ when $\Delta$ grounds A and nothing needs to be added to $\Delta$ to get a fully satisfactory explanation of A. One way to ensure that nothing needs to be added is to require that everything that might be added is already included. This suggest the following definition:

(F1) $\Delta$ fully grounds $\mathrm{A}=_{\text {def. }}$ (i) $\Delta$ partially grounds $\mathrm{A}$; and (ii) for any $\Gamma$ distinct from $\Delta$ that partially grounds $\mathrm{A}, \Gamma$ is a proper sub-collection of $\Delta$.

If, however, there could be NAG cases of either of the two sorts reviewed above, this condition is insufficient. In any such case, the collection of all the partial grounds will still fail to be a full ground. In addition, F1 also fails to identify a necessary condition. Suppose that the following three facts obtain:

BURGUNDY: The ball is burgundy.

RED: The ball is red.

COLOR: The ball is colored.

We take it that BURGUNDY fully grounds COLOR, as does RED. Given F1, however, neither does. According to F1, for BURGUNDY to count as a full ground for COLOR, every partial ground of COLOR must be included in it—so, RED must be included, as RED is at least a partial ground. But, of course, RED is not included given that BURGUNDY is a collection of one, so BURGUNDY fails to be a full ground for COLOR. 
The ground in this case that is "left out" is itself part of a chain of grounds from BURGUNDY to COLOR, not something that augments BURGUNDY in its grounding of COLOR. This suggests a natural fix:

(F2) $\Delta$ fully grounds $\mathrm{A}=_{\text {def. }}$ (i) $\Delta$ partially grounds A; and (ii) for any $\Gamma$ distinct from $\Delta$ that partially grounds $A$, either $\Gamma$ is a proper sub-collection of $\Delta, \Gamma$ is a partial ground of some fact among $\Delta$, or $\Delta$ is a partial ground of some fact among $\Gamma$.

The conditions in F2 are still too strong. Suppose the following facts obtain:

BASIL: Basil is a goldfish.

CLARA: Clara is a goldfish.

GOLDFISH: Something is a goldfish.

As before, we have two facts (BASIL, CLARA) both of which are full grounds for a single fact (GOLDFISH). In this case, however, the two full grounds are not part of a single grounding chain; they are independent of each other. This independence makes it clear why an appeal to exhaustiveness is on the wrong track. GOLDFISH is fully grounded twice over, and this is entirely unproblematic.

Instead of looking outside $\Delta$ for grounds that may be left out, let us look to the relation between $\Delta$ and $A$. One strategy —as seen in discussions of explanation generally - is to require that the explanatory fact suffice for the explained fact.

(F3) $\Delta$ fully grounds $\mathrm{A}=_{\text {def. }}$ (i) $\Delta$ partially grounds A; and (ii) necessarily, if $\Delta$ obtains, then A obtains.

This proposal is plainly too crude, however, as conditions (i) and (ii) are too easy to satisfy. Consider this fact:

BASIL \& SUM: Basil is a goldfish and $2+2=4$. 
BASIL satisfies the two conditions of F3. It partially grounds BASIL \& SUM simply by being the first conjunct thereof, and it is of course necessary that if Basil is a goldfish, then Basil is a goldfish and $2+2=4$. If sufficiency is part of the answer here, we must ensure that the sufficiency is relevant to the fact that is fully grounded.

The natural suggestion is to appeal to the essences of the relevant entities, where the relevant notion of essence is the intuitive one that Fine (1995: 281) calls "immediate constitutive essence". The fact that BASIL suffices for BASIL \& SUM is obviously not part of the essence of, say, addition or being a goldfish. Consider, then, F4:

(F4) $\Delta$ fully grounds $\mathrm{A}=_{\text {def. }}$ (i) $\Delta$ partially grounds A; and (ii) it's essential to constituents of either A or facts among $\Delta$ (or collections of such entities) that if $\Delta$ obtains then A obtains.

Unfortunately, F4 is too weak. Consider the following facts:

CONVICTED: Desmond is a rightly convicted thief.

STOLEN: Someone's possessions were stolen.

CRIMINAL: Desmond is a criminal.

Proposal F4 implies that CONVICTED is a full ground of the conjunctive fact STOLEN \& CRIMINAL. First, CONVICTED partially grounds STOLEN \& CRIMINAL, since it fully grounds the second conjunct thereof. Second, it's necessary that if Desmond is a convicted thief then someone's possessions were stolen and Desmond is a criminal. Third, and finally, it seems to lie in the nature of the relevant entities - the property of being a rightly convicted thief, for instance-that it does so. It is because of what it is to be a rightly convicted thief that if it is instantiated, someone's possessions were stolen and the person who instantiates it is a criminal. Yet CONVICTED certainly does not fully ground STOLEN \& CRIMINAL. The fact that someone's possessions were stolen partially explains why Desmond is a rightly convicted thief, not the other way around. 
It may help to have another counterexample with a similar structure. Consider the following facts:

BEIJING: Beijing exists.

SINGLETON: $\{$ Beijing $\}$ exists.

SET: There exists a set.

Proposal F4 implies that SINGLETON is a full ground of the conjunctive fact BEIJING \& SET. First, SINGLETON is a full ground of the fact that there is a set and thus a partial ground of BEIJING \& SET. Second, it's necessary that if $\{$ Beijing $\}$ exists, then Beijing exists and there is a set. Third, of course, it lies in the nature of \{Beijing\} that if $\{$ Beijing exists, then Beijing exists and there is a set. But it would be an error to say that SINGLETON fully grounds BEIJING \& SET. The fact that $\{$ Beijing $\}$ exists helps explain the second conjunct, but not the first. The fact that Beijing exists helps explain why $\{$ Beijing\} exists, but certainly not the reverse.

Perhaps we should strengthen the required essential conditional truth to include grounding as follows:

(F5) $\Gamma$ fully grounds $\mathrm{A}=_{\text {def. }}$ (i) $\Delta$ partially grounds $\mathrm{A}$; and (ii) it's essential to constituents of either A or facts among $\Delta$ (or collections of such entities) that if $\Delta$ obtains then $\Delta$ partially grounds A.

F5, however, also counts SINGLETON as a full ground of BEIJING \& SET. It lies in the nature of $\{$ Beijing $\}$ that if $\{$ Beijing $\}$ exists, then Beijing exists and there exists some set or other. It is no less plausible to count it as an essential truth about \{Beijing\} that if \{Beijing\} exists, then that fact partially grounds that conjunctive fact-after all, it seems right to say that part of the nature of $\{$ Beijing $\}$ is that its existence grounds the existence of a set. The example of thieving Desmond can also be put to the same purpose. 


\section{How to define full grounding in terms of partial grounding}

Whatever full grounding is, it is supposed to underwrite a kind of fully satisfactory explanation. In citing the full grounds, you give an explanation of the grounded fact where that explanation is in some sense complete. We think the relevant sense of completeness is exemplified in the following inference:

1. The fact that the ball is burgundy fully grounds the fact that the ball is red.

2. Therefore, the fact that the ball is red is nothing over and above the fact that the ball is burgundy.

We think this inference is valid. If $\mathrm{A}$ is fully grounded by $\Delta$, then $\mathrm{A}$ is nothing over above $\Delta$. There's nothing more to $\mathrm{A}$ than those facts. This is why $\mathrm{A}$ is completely explained: in citing $\Delta$, we cite enough to ensure that the entirety of $\mathrm{A}$ is grounded in other facts—nothing about what it takes for A to obtain is left unexplained.

At least as we understand grounding talk, if $\mathrm{A}$ is fully grounded by $\Delta$, then $\mathrm{A}$ is in a sense "reducible" to the facts collected in $\Delta$. This is a sense of reduction that does not require identity; intuitively, the idea is of containment - the fully grounded fact is contained already within the facts that fully ground it. The idea that full grounding licenses a "nothing over and above" conclusion is certainly not original to us. A number of grounding theorists have ventured claims of this sort, often describing the grounded fact as an "ontological free lunch" or as "constituted by" the grounding facts. ${ }^{5}$ This implication of full grounding seems to us a clue as to how full grounding should be understood. The way full grounding entails that the grounded is nothing over and above the grounds exbibits what it is that full grounding is supposed to do-yield a fully satisfactory explanation of the grounded fact. It thus seems a good place to look for a definition of full grounding.

We expect, of course, that some readers will find worrisome any attempt to define full grounding in a way that makes unabashed use of the "nothing over and above"

5 See Fine 2001: 15-16; Fine 2012: 39; Schaffer 2009: 353; for dissent, cf. Audi 2012: 708; deRosset 2013b. 
locution. We reject the claim that "nothing over and above" is too obscure for theoretical work. We admit, of course, that it is not a phrase made familiar and safe through any well-established regimentation. And it would not be surprising if some genuine semantic indeterminacy accompanies actual use of the phrase. These points hardly undermine its suitability as a tool in defining full grounding. Not to put too fine a point on it, the same points could have been made about those phrases that signal the notion of grounding. An enthusiast for grounding who bristles at the alleged obscurity of "nothing over and above" owes us an explanation of how that phrase is worse off than "in virtue of," "makes it the case," or the like.

In addition, however, there are several points to be made in defense of using this locution in theoretical work. (We will hereafter speak of the "NOA" locution or notion expressed thereby.) We stress three such points. First, the NOA locution or cognates thereof can be found both in philosophical work and in ordinary English. While "nothing over and above" is not so common in ordinary English, "nothing but" and "nothing more than" are not unusual. The idea is certainly not just a philosopher's invention. And in philosophy itself, one can find it being used in a wide variety of areas both today and in work dating back many decades. Here is an example from Wisdom nearly a century ago:

We must not say that a table is the string of events which make up its life-history. For then to say of a table that it collapsed is to say of a string of events that they collapsed. On the other hand, the fact that the table collapsed is perhaps nothing but the fact that a set of suitably inter-related events includes a collapse (Wisdom 1931: 192).

Note that the "nothing but" claim here is combined with the denial of identityprecisely the feature of "nothing over and above" talk that is most likely to cause consternation. ${ }^{6}$ We want to stress this point, as we have also linked the NOA notion

\footnotetext{
${ }^{6}$ For another good example, consider Wiggins' (1968: 91) claim from just half a century ago that, while a tree is distinct from the molecules that compose, it is "not something over and above" those molecules. Notably, Wiggins explicitly draws as a moral the claim that "[o]ver and above is one question, identity is another" (92).
} 
to "reduction," which is sometimes understood as a relation that requires identity. As we understand the NOA locution, one way for a fact A to be nothing over and above a fact $\mathrm{B}$ is for $\mathrm{A}$ and $\mathrm{B}$ to be identical-but it is not the only way. The fact that the ball is red might be nothing over and above and the fact that it is burgundy even though the constituents of these two facts are not identical (the property of being red is not identical with that of being burgundy). What matters is that whatever it takes for A to obtain is built into the obtaining of $\mathrm{B}$. In this sense, we think, it is uncontroversial that physicalism must be reductive-mental facts must be in this way built into the physical facts, regardless of whether they are identical with any such facts.

Second, to get at the NOA notion we need not rely solely on the "nothing ..." phrases. Supplementary glosses are available that are, we believe, in keeping with the use of the NOA locution in the literature. As noted above, the idea here is intuitively that of a kind of containment. Where $\mathrm{A}$ is nothing over and above $\Delta, \mathrm{A}$ is in some sense already there within $\Delta$. It is not merely necessary that A obtain when $\Delta$ obtains; rather, $\mathrm{A}$ is built into $\Delta$ so that a world in which $\Delta$ obtains is "already thereby" a world in which A obtains (Goff 2017: 43).

So long as the relata invoked in using the NOA locution are facts or collections of facts, another gloss seems especially helpful. Recall what Jackson has described as a "location problem": the task of seeing how certain truths not spelled out explicitly in some description of a situation are nonetheless implicit in that description (1998: 15). For a simple example, the fact that the pixels are arranged in such-and-such a way might implicitly include the fact that the screen depicts a circular figure. It seems to us plain that if this notion of implicit content is clear enough, then so is the NOA notion; indeed, they seem identical so long as NOA is used to relate facts. And-we stress-our own use of the NOA notion is limited to cases where the relata are facts. By endorsing the claim that what is fully grounded is nothing over and above the grounds we don't need to rely on talk of an object or a property as nothing over and above other objects or properties; our reliance on the NOA locution is in this way rather minimal. 
Let us review one further way of getting a handle on the NOA locution. This way appeals to the notion of ontological commitment. Lewis claims that mereology is ontologically innocent in the following sense: "In general, if you are already committed to some things you incur no further commitment when you affirm the existence of their fusion" (1991: 81-82). Repurposing the proposal for facts, we may suggest a general guide: whenever one fact is nothing over and above another, one who is already committed to the former fact obtaining does not, in acknowledging that the latter also obtains, incur a further commitment. While we think this is a helpful way to get a grip on the NOA notion, we caution against too blunt a use of it. Is a "further commitment" to be understood as merely any commitment to some fact distinct from any to which you were previously committed? If so, the guide delivers the wrong results. While RED is nothing over and above BURGUNDY in the relevant sense, these are distinct facts, given that they involve distinct properties. Compare the present point to what Chalmers says about the biological facts when he supposes them to be a free lunch with respect to the physical facts: "They may be different facts (a fact about elephants is not a microphysical fact), but they are not further facts" (1996: 41). The "further" here is akin to how the term is used in Lewis's discussion-to have a further commitment in the relevant sense requires that the commitment have an additional cost with respect to one's other commitments, which is potentially relevant to the ontological economy of one's theory compared to others (Cameron 2014; Schaffer 2015). In accepting RED in addition to BURGUNDY one is not sticking one's neck out more than one already has.

The appeal to ontological commitment may be most useful in getting at the relevant NOA notion if it is combined with the talk of containment. If, after accepting RED one comes to feel that one has stuck one's neck out further than one would like, that either means one thinks RED is not contained in BURGUNDY or that one has yet to reckon with the costs of accepting BURGUNDY in the first place. The point, in any case, is that the appeal to commitment can indeed be useful in triangulating the NOA notion, especially if used in combination with other ways of getting a handle on the idea. 
Finally, as a third point about our use of the NOA locution, even if ideally we should dispense with NOA talk, so long as its use consistently tracks a coherent notion, we can at least arrive at a true claim about the necessary and sufficient conditions for a ground to count as a full ground. We think that the proposal we will offer below is both extensionally adequate and illuminating in that it gets at what makes a ground a full ground. But if we are wrong about the latter point, the proposal can still serve as a constraint on further theorizing about grounding. It is hard to believe that the NOA locution is in such poor shape it cannot even serve to circumscribe a constraint of this sort.

A different objection to our use of the NOA locution is not that it is too obscure but that it is too close in meaning to the notion of full grounds to be used in illuminating the latter. We agree they are closely related, but there is some significant distance between them. Note that nothing over-and-above-ness has different formal properties than grounding. Grounding is apparently irreflexive and asymmetric. Since one way for $\mathrm{A}$ to be nothing over and above $\mathrm{B}$ is for $\mathrm{A}$ and $\mathrm{B}$ to be the same fact, nothing-over-and-above-ness in the relevant sense is by contrast reflexive. And, while nothing-over-and-above-ness is not symmetric (STOLEN is nothing over and above CONVICTED but not vice versa), it's not asymmetric either (BEIJING is nothing over and above SINGLETON and vice versa).

(We acknowledge, though, that both grounding and nothing-over-and-above-ness are transitive: if $\mathrm{A}$ is nothing over and above $\mathrm{B}$, and $\mathrm{B}$ is nothing over and above $\mathrm{C}, \mathrm{A}$ is nothing over and above $\mathrm{C}$. To revert to the containment language: if all it takes for A to obtain is contained in $\mathrm{B}$, and all it takes for $\mathrm{B}$ to obtain is contained in $\mathrm{C}$, then this consequence is inevitable.)

The distance between the notions is illustrated by the fact that one cannot easily provide a simple definition of full grounding in terms of NOA. Consider the following proposal:

(F6) $\Delta$ fully grounds $\mathrm{A}=_{\text {def. }}$ (i) $\mathrm{A}$ is nothing over and above $\Delta$; and (ii) $\mathrm{A}$ is not identical with any fact among $\Delta$. 
Our example of thieving Desmond shows that F6 is insufficient. If we take CONVICTED as the ground and STOLEN \& CRIMINAL as the grounded, the example meets the conditions in F6. However exactly facts are to be individuated, it seems a mistake to treat CONVICTED as identical with STOLEN \& CRIMINAL. Moreover, the latter isn't among the former, as the former is a collection of one. Further, STOLEN \& CRIMINAL seems to be nothing over and above CONVICTED. To say otherwise would imply that the conjunctive fact is indeed something "over and above" CONVICTED, that there is something to this fact that goes beyond what is already built into CONVICTED. And that's a mistake. First, there being someone whose possessions were stolen is nothing over and above Desmond's being a rightly convicted thiefSTOLEN is built into CONVICTED, even if it's not grounded by such. Once we have on the scene, so to speak, the fact that Desmond is a rightly convicted thief, we already have on the scene the fact that someone's possessions were stolen. Second, Desmond's being a criminal is nothing over and above Desmond being a rightly convicted thief; this looks like a simple case of full grounding with a nothing-overand-above consequence. Yet CONVICTED is not a full ground of STOLEN \& CRIMINAL.

We have seen that F6 is not extensionally adequate. You might think that it fails in another way as well: should we not say that $\mathrm{A}$ is nothing over and above $\Delta$ because $\Delta$ fully grounds $A$, not that $\Delta$ fully grounds $\mathrm{A}$ because $\mathrm{A}$ is nothing over and above $\Delta$ ? This point may make it seem that appealing to "nothing over and above" in defining full grounds is simply on the wrong track.

But this would be premature. Suppose that $\Delta$ fully grounds $\mathrm{A}$. In this case, $\mathrm{A}$ is nothing over above $\Delta$, and we agree that this is due to $\Delta$ 's role in grounding $\mathrm{A}$-but this role can be characterized as partial grounding. We don't need to say that A is nothing over and above $\Delta$ in virtue of the fact that $\Delta$ is a full ground of $A$; we can say instead that $\mathrm{A}$ is nothing over and above $\Delta$ because of facts about $\Delta$ 's partial grounding of $A$. Otherwise put: $\mathrm{A}$ is nothing over and above $\Delta$ because of $\Delta$ 's partial grounding of A; this then is why $\Delta$ fully grounds A. In slogan form: 
Full grounding is what happens when partial grounding works in a way that ensures that the grounded is nothing over and above the grounds.

How might this idea yield a definition? Our slogan invokes three conditions. The first is that $\Delta$ partially grounds $\mathrm{A}$; the second is that $\mathrm{A}$ is nothing over and above $\Delta$; and the third concerns the relationship between these two. To follow our slogan, we want to specify that relation by spelling it out as clause (iii) below:

$\Delta$ fully grounds $\mathrm{A}={ }_{\text {def. }}$ (i) $\Delta$ partially grounds $\mathrm{A}$; (ii) $\mathrm{A}$ is nothing over and above $\Delta$; and (iii) $\ldots$

A natural attempt is to appeal to sufficiency again:

Necessarily, if $\Delta$ obtains, then $\mathrm{A}$ is nothing over and above $\Delta$.

This won't do, however. The account so formulated implies that SINGLETON fully grounds BEIJING \& SET. Condition (i) is met. SINGLETON fully grounds SET, so it partially grounds the conjunction of which SET is a conjunct. Condition (ii) is met as well. This may seem less obvious at first, but we can see the point by considering the contrary option, that BEIJING \& SET is indeed something over and above SINGLETON. That claim is surely false: once \{Beijing\} exists, everything that makes for the conjunctive fact is already there on the scene. The existence of Beijing is built into the existence of $\{$ Beijing $\}$ and the existence of at least one set is also built into the existence of $\{$ Beijing\}. Finally, the third condition is met, as there is no world in which SINGLETON obtains and the previous two points don't apply.

Grounding- and essence-theoretic approaches to the connection between the fact that $\mathrm{A}$ is nothing over and above $\Delta$ and the fact that $\Delta$ partially grounds $\mathrm{A}$ likewise fail, though we won't go into all the details here. Consider just one attempt; say we spell out the third condition as follows:

Necessarily, if $\Delta$ partially grounds $\mathrm{A}$, then $\mathrm{A}$ is nothing over and above $\Delta$ and $[\Delta$ partially grounds $\mathrm{A}$ ] itself partially grounds [A is nothing over and above $\Delta$ ]. 
The SINGLETON and BEIJING \& SET case works as a counterexample here as well. It seems that the fact that SINGLETON partially grounds BEIJING \& SET itself partially grounds the fact that BEIJING \& SET is nothing over and above SINGLETON. This is because SINGLETON partially grounds BEIJING \& SET by fully grounding the second conjunct, which ensures that the second conjunct is nothing over and above SINGLETON. The same point can be made using the CONVICTED and STOLEN \& CRIMINAL case.

We propose instead that the relevant connection is the NOA relation itself. The thought is that when $\Delta$ fully grounds $\mathrm{A}$, not only is A nothing over and above $\Delta$; further, this status of $\mathrm{A}$ is itself nothing over and above the fact that $\Delta$ partially grounds A. This is the last step in devising our proposed definition-the "Iterated Nothing Over and Above" definition:

(INOA) $\Delta$ fully grounds $\mathrm{A}={ }_{\text {def. }}$ (i) $\Delta$ partially grounds $\mathrm{A}$; (ii) $\mathrm{A}$ is nothing over and above $\Delta$; and (iii) [A is nothing over and above $\Delta$ ] is itself nothing over and above $[\Delta$ partially grounds $\mathrm{A}]$.

The definition boasts a pleasing symmetry of structure: we have full grounding when we have partial grounding (the first condition) and the grounded is nothing over and above the grounds (the second condition) and when the second condition is itself nothing over and above the first condition (the third condition). More important, however, is that the definition is motivated by the idea that full grounding is what we get when partial grounding works in a way to make the grounded nothing over and above the grounds. So far as INOA reflects this rationale, it has considerable plausibility.

INOA must also, of course, classify cases appropriately. Let us consider how it fares with respect to a modest budget of varying examples. For our first example, the full grounding claim to be vindicated is (1):

(1) BEIJING fully grounds SINGLETON.

According to INOA, (1) is true if and only if: 
(1a) BEIJING partially grounds SINGLETON.

(1b) SINGLETON is nothing over and above BEIJING.

(1c) [SINGLETON is nothing over and above BEIJING] is itself nothing over and above [BEIJING partially grounds SINGLETON].

Supposing that singletons are fully grounded by their members, (1a) is met. While the commitment to BEIJING obtaining and the commitment to SINGLETON obtaining are distinct commitments, plausibly the latter doesn't have an additional cost to the former (Cameron 2014: 95). Given our discussion of the connection between NOA and commitment above, this suggests that (1b) is met. Why think that (1c) is also correct? We will offer a way of thinking about this that is — we freely admitsomewhat loose and intuitive. Nonetheless, it seems to us to be on the right track, and what matters here is that the comments below make (1c) plausible, even if there are good questions about how the comments below themselves should be elaborated.

Intuitively, there is nothing more to [SINGLETON is nothing over and above BEIJING] than [BEIJING partially grounds SINGLETON] because of the way the former partially grounds latter. We understand talk of "the way" in which BEIJING partially grounds SINGLETON in terms of certain features of these facts. Given the character of the relata here, there is nothing more to this fact (SINGLETON) being nothing over and above that fact (BEIJING) than that fact partially grounding this fact. The grounding relation itself does not ensure this, as it is neutral between full and merely partial grounding. But when it relates the appropriate relata, the status of the grounded as nothing over and above the grounding is itself a free lunch.

Not just any features of those specific relata matter, of course. Consider a feature of the relata that isn't relevant to BEIJING's partially grounding SINGLETON, such as BEIJING's being such that it is partially grounded by facts concerning certain social conventions. This feature isn't relevant to the truth of the iterated NOA claim either. But now consider features of the relata that are relevant to BEIJING's partially grounding SINGLETON, such as BEIJING's being such that it concerns the existence of 
an urelement, and SINGLETON's being such that it concerns the existence of a set, one with that urelement as its sole member. These sorts of features are relevant to the truth of the iterated NOA claim.

For another example of full grounding, consider facts about knowledge again; but this time let us suppose that the fact that a person knows something can be analyzed into the claim that the person has an undefeated, true, justified belief with that content. Let us use again Amy's knowledge as our example:

KNOWLEDGE: Amy knows that humans have set foot on the moon.

BELIEF: Amy has an undefeated, true, justified belief that humans have set foot on the moon.

If indeed knowledge can be analyzed in this way, it seems safe to say that

(2) BELIEF fully grounds KNOWLEDGE.

According to INOA, (2) is true if and only if:

(2a) BELIEF partially grounds KNOWLEDGE.

(2b) KNOWLEDGE is nothing over and above BELIEF.

(2c) [KNOWLEDGE is nothing over and above BELIEF] is itself nothing over and above [BELIEF partially grounds KNOWLEDGE].

It seems that INOA handles this case well. As before, it is the third condition that deserves special note. Recall that we are here stipulating that knowledge in general is just a matter of having an undefeated justified true belief. The way BELIEF partially grounds KNOWLEDGE is by exhibiting the particular person (Amy) and proposition (that humans have set foot on the moon) as being an instance of that generalization. That generalization itself may be understood as a kind of "nothing over and above" claim: generally, knowing that $\mathrm{p}$ is nothing more than having an undefeated justified true belief that $\mathrm{p}$. If that is right, then the way that BELIEF partially grounds KNOWLEDGE is by relating the particular case of Amy and Amy's belief to that 
general fact about what knowledge generally "boils down" to. As a result, the fact that KNOWLEDGE is nothing over and above BELIEF is already built into the fact that BELIEF partially grounds KNOWLEDGE. So (2c) is true, as predicted by INOA.

Let us now turn to an example that caused trouble before. Consider the false (3):

(3) CONVICTED fully grounds STOLEN \& CRIMINAL.

According to INOA, (3) is true if and only if:

(3a) CONVICTED partially grounds STOLEN \& CRIMINAL.

(3b) STOLEN \& CRIMINAL is nothing over and above CONVICTED.

(3c) [STOLEN \& CRIMINAL is nothing over and above CONVICTED] is itself nothing over and above [CONVICTED partially grounds STOLEN \& CRIMINAL].

We have already discussed (3a) and (3b) and why they should be accepted. We submit that (3c) should be rejected. To see why, it helps to attend to the way in which a fact may serve as a ground of another. The way that CONVICTED partially grounds STOLEN \& CRIMINAL is by means of two steps: first, CONVICTED fully grounds CRIMINAL. The fact that Desmond is a rightly convicted thief fully explains the fact that he is a criminal. Second, however, as CRIMINAL is one conjunct of STOLEN \& CRIMINAL, CONVICTED also serves as a merely partial ground of that conjunctive fact. In this light, the fact that CONVICTED partially grounds STOLEN \& CRIMINAL doesn't reflect on the status of its first conjunct STOLEN. For something to explain the nothing-over-and-above status of the conjunctive STOLEN \& CRIMINAL, however, it obviously must illuminate the status of both conjuncts.

When we argued earlier that (3b) is correct, we emphasized that STOLEN is built into CONVICTED: for it to be the case that Desmond is a rightly convicted thief $i$ in part for him to have stolen something, and for him to have stolen something is in part for someone's possessions to have been stolen. If we are to identify something that contains the entirety of the fact that STOLEN \& CRIMINAL is nothing over and above CONVICTED, we must find something that includes this fact about the way STOLEN is 
built into CONVICTED. The fact that CONVICTED partially grounds STOLEN \& CRIMINAL fails on this count. As we might put it, (3b) is indeed something over and above (3a); that additional something is, precisely, the fact that STOLEN is nothing over and above CRIMINAL. A similar story can be told about the case of SINGLETON as an alleged full ground of BEIJING \& SET.

For one last example, we consider a new case that, we think, illustrates the utility of INOA in adjudicating new and odd cases. Suppose again that BURGUNDY (the fact that the ball is BURGUNDY) obtains. In this case, RED (the fact that the ball is red) obtains as well. What should we say about (4)?

(4) BURGUNDY fully grounds BURGUNDY \& RED.

We imagine that you hesitate a bit (we certainly have), but with INOA as a guide, we can identify what seem to be the relevant considerations and arrive at a satisfying answer. Given INOA, (4) is true if and only if:

(4a) BURGUNDY partially grounds BURGUNDY \& RED.

(4b) BURGUNDY \& RED is nothing over and above BURGUNDY.

(4c) [BURGUNDY \& RED is nothing over and above BURGUNDY] is itself nothing over and above [BURGUNDY partially grounds BURGUNDY \& RED].

It is obvious that (4a) is correct. But the way BURGUNDY partially grounds BURGUNDY \& RED is of special interest, since there is a sense in which it does this twice over. BURGUNDY does it first by supplying one conjunct of the conjunctive fact, namely BURGUNDY. By itself, that's enough for BURGUNDY to be a partial ground. But it is a partial ground of the conjunctive fact again by being a full ground of the other conjunct, namely RED. The fact that BURGUNDY partially grounds BURGUNDY \& RED involves the former relating to the latter in both of these ways.

It also seems clear that (4b) is correct. If BURGUNDY \& RED were something over and above BURGUNDY, it seems that at least one of its conjuncts must be something over and above BURGUNDY. That could hardly be the case with the first conjunct, as that 
is BURGUNDY itself, and the second conjunct, RED, is nearly a paradigm case of one fact being nothing over and above another one.

But how is (4c) to be assessed? The points made above in justifying both (4a) and (4b) are at this point helpful, as they point to features of both that seem relevant to (4c). Let us ask about (4b): what is involved in the fact that BURGUNDY \& RED is nothing over and above BURGUNDY? In motivating (4b), we made salient two features of the situation. The first was the identity of one conjunct of BURGUNDY \& RED with BURGUNDY itself. Does that fact involve anything over and above what is involved in the partial grounding fact (4a)? No. As we saw, one of the two ways in which the partial grounding of BURGUNDY \& RED by BURGUNDY works is by means of the identity of the latter with one conjunct of the former. The first salient feature of (4b) is thus not anything over and above the partial grounding fact (4a).

The second point about (4b) highlighted by our comments above was that RED is nothing over and above BURGUNDY. Is that fact anything over and above the partial grounding fact (4a)? Again, no. One of the two ways in which the partial grounding of BURGUNDY \& RED by BURGUNDY works is by means of the latter fully grounding RED. That fact itself involves - as per INOA — the fact that RED is nothing over and above BURGUNDY. The nothing-over-and-above status of BURGUNDY \& RED then, is yet again not anything more than what is involved in the partial grounding fact (4a).

Together these points make (4c) plausible, and if INOA is correct, (4) itself is true. Is that verdict plausible? When we consider (4) without any suggested account of full grounding, it is hard to know what to say. Working through how INOA applies to this case not only delivers the verdict that (4) is true, however; it also helps make (4) seem intuitively correct—or so it seems to us.

Our brief review of cases is far from dispositive, of course. There remains much more one could do to investigate the adequacy of the INOA account by way of its treatment of cases. Nonetheless, given the plausibility of the slogan that guided our 
formulation of INOA, we suggest INOA as a serious contender for a definition of full grounding. ${ }^{7}$

\section{References}

Armstrong, D. M. (2004) Truth and Truthmakers. Cambridge: Cambridge University Press.

Audi, P. (2012) 'Grounding: toward a theory of the in-virtue-of relation'. Journal of Philosophy, 109, 685-711.

Cameron, R. (2014) 'Parts generate the whole, but they are not identical to it'. In A. J. Cotnoir and D. Baxter (eds.), Composition as Identity (Oxford: Oxford University Press), pp. 90-107.

Chalmers, D. (1996) The Conscious Mind. Oxford: Oxford University Press.

Chudnoff, E. (2013) Intuition. Oxford: Oxford University Press.

Clark, M. (2015) 'A puzzle about partial grounding'. Thought, 4, 189-197.

deRosset, L. (2013a) ‘What is weak ground?' Essays in Philosophy, 14, 7-18.

deRosset, L. (2013b) 'No free lunch'. In M. Hoeltje et al (eds.), Varieties of Dependence (Munich: Philosophia Verlag), pp. 243-270.

Fine, K. (2001) 'The question of realism'. Philosophers' Imprint, 1, 1-30.

Fine, K. (2012) 'Guide to ground'. In F. Correia \& B. Schnieder (eds.), Metaphysical Grounding: Understanding the Structure of Reality (Cambridge: Cambridge University Press), pp. 37-80.

Goff, P. (2017) Consciousness and Fundamental Reality. Oxford: Oxford University Press Jackson, F. (1998) From Metaphysics to Ethics. Oxford: Oxford University Press.

\footnotetext{
7 We presented parts of this paper at Lund University, UMass Amherst, University of Geneva, and University of Gothenburg. Thanks to our audience members for the helpful feedback. We wish to thank Ricki Bliss, Darragh Byrne, Claudio Calosi, Fabrice Correia, Scott Dixon, Jeff Dunn, Kit Fine, Dan Gibberman, David Kovacs, Jon Litland, Anna-Sofia Maurin, Neil Mehta, Chris Meacham, Kevin Mulligan, Donnchadh O'Connaill, Sven Rosenkranz, Maria Scarpati, Ted Sider, Alex Skiles, Robin Stenwall, Naomi Thompson, and Nathan Wildman. Finally, thanks are due to two anonymous referees at this journal.
} 
Kim, J. (2009) 'Supervenient and yet not deducible': Is there a coherent concept of emergence?' In A. Hieke and H. Leitgeb (eds.), Reduction: Between the Mind and the Brain (Frankfurt: Ontos Verlag), pp. 53-72.

Leuenberger, S. (2020) 'The fundamental: ungrounded or all-grounding?’ Philosophical Studies, 177, 2647-2669.

Lewis, D. (1991) Parts of Classes. Oxford: Wiley-Blackwell.

Litland, J. E. (2015) 'Grounding, explanation, and the limit of internality'. Philosophical Review, 124, 481-532.

Rosen, G. (2010) 'Metaphysical dependence: grounding and reduction'. In B. Hale and A. Hoffman (eds.), Modality: Metaphysics, Logic, and Epistemology (Oxford: Oxford University Press), pp. 109-136.

Rosen, G. (2015) 'A puzzle postponed'. Thought, 4, 198-201.

Schaffer, J. (2009) 'On what grounds what'. In D. Chalmers et al (eds.), Metametaphysics (Oxford: Oxford University Press), pp. 347-383.

Schaffer, J. (2015) 'What not to multiple without necessity'. Australasian Journal of Philosophy, 93, 644-664.

Schaffer, J. (2016) 'Grounding in the image of causation'. Philosophical Studies, 173, 49100.

Skiles, A. (2015) 'Against grounding necessitarianism’. Erkenntnis, 80, 717-751.

Werner, J. (forthcoming) 'A minimality constraint on grounding'. Erkenntnis.

Wiggins, D. (1968) 'On being in the same place at the same time'. Philosophical Review, 77, 90-95.

Williamson, T. (2000) Knowledge and its Limits. Oxford: Oxford University Press.

Wilson, J. (2018) ‘Grounding-based formulations of physicalism’. Topoi, 37, 495-512.

Wisdom, J. (1931) 'Logical constructions I'. Mind, 40, 188-216. 